\title{
Measurements of self-guiding of ultrashort laser pulses over long distances
}

\author{
K. Poder ${ }^{1,2}$, J. M. Cole ${ }^{1}$, J. C. Wood ${ }^{1}$, N. C. Lopes ${ }^{1,3}$, S. Alatabi ${ }^{1}$, P. S. Foster ${ }^{4}$, C. Kamperidis ${ }^{1,5}$, O. Kononenko ${ }^{2}$, \\ C. A. Palmer ${ }^{2}$, D. Rusby ${ }^{4}$, A. Sahai ${ }^{1}$, G. Sarri ${ }^{6}$, D. R. Symes ${ }^{4}$, J. R. Warwick ${ }^{6}$, S. P. D. Mangles ${ }^{1}$, and Z. Najmudin ${ }^{1 \dagger}$ \\ 1 The John Adams Institute for Accelerator Science, \\ Imperial College, London, SW7 2BZ, United Kingdom \\ 2 Deutsches Elektronen-Synchrotron DESY, D-22607 Hamburg, Germany \\ ${ }^{3}$ GoLP/Instituto de Plasmas e Fusão Nuclear, Instituto Superior Tecnico, 1049-001 Lisboa, Portugal \\ ${ }^{4}$ Central Laser Facility, Rutherford Appleton Laboratory, Didcot, OX11 0QX, United Kingdom \\ ${ }^{5}$ ELI-ALPS, ELI-HU Non-Profit Ltd, H-6720 Szeged, Dugonics tr 13, Hungary and \\ ${ }^{6}$ Queen's University Belfast, Belfast, BT7 1NN, United Kingdom
}

(Dated: September 13, 2017)

\begin{abstract}
We report on the evaluation of the performance of self-guiding over extended distances with $f / 20$ and $f / 40$ focussing geometries. Guiding over $39 \mathrm{~mm}$ or more than 100 Rayleigh ranges was observed with the $f / 20$ optic at $n_{e}=1.5 \times 10^{18} \mathrm{~cm}^{-3}$. Analysis of guiding performance found that the extent of the exiting laser spatial mode closely followed the matched spot size predicted by 3D non-linear theory. Self-guiding with an f/40 optic was also characterised, with guided modes observed for a plasma length of $90 \mathrm{~mm}$ and a plasma density of $n_{e}=9.5 \times 10^{17} \mathrm{~cm}^{-3}$. This corresponds to self-guided propagation over 53 Rayleigh ranges and is similar to distances obtained with discharge plasma channel guiding.
\end{abstract}

\section{INTRODUCTION}

Laser plasma acceleration, which started as a theoretical concept nearly four decades ago [1], has developed into a multifaceted research field with potential to become a source of high energy electron beams in the future [2]. These compact accelerators make use of accelerating gradients of the order of $100 \mathrm{GV} \mathrm{m}^{-1}$ and have been shown to generate relativistic monoenergetic electron beams in millimetre distances [3-5]. The short-pulse nature of the generated electron beam along with its inherent synchronisation to a laser pulse has allowed development of bright compact, femtosecond photon sources [6-9] as well as demonstration of relativistic, neutral leptonic plasmas [10].

Laser wakefield accelerators employ as an accelerating structure a relativistically moving, ion rich plasma cavity travelling in the wake of an intense laser pulse. This wake is set up after electrons expelled from regions of high intensity by the laser's ponderomotive force return to their initial positions, forming an electron plasma wave. For high intensity lasers, where the normalised vector potential $a_{0}=e E_{0} /\left(m_{e} \omega_{0} c\right)$ approaches unity and beyond, this wake can become highly non-linear with peak accelerating fields of $\sqrt{a_{0}} m_{e} c \omega_{p} / e$ inside the cavity [11], where $\omega_{p}$ is plasma electron frequency and $\omega_{0}$ is the angular frequency of the laser. The production of high electron beam energies requires acceleration distances longer than the Rayleigh range of the laser. The Rayleigh range $z_{R}=\pi w_{0}^{2} / \lambda_{0}$, where $w_{0}$ is the $1 / e$ radius of the electric field of the focus and $\lambda_{0}=2 \pi c / \omega_{0}$ is the laser wavelength, is a characteristic distance over which the laser spot size increases by a factor of $\sqrt{2}$ leading to halving

\footnotetext{
* k.poder12@imperial.ac.uk

$\dagger$ zn1@imperial.ac.uk
}

the intensity of the laser. Hence, in order to achieve long acceleration distances the laser diffraction must be countered by some form of guiding.

Indeed, to obtain higher output energies, wakefield accelerators need to be operated at lower plasma densities, such that they have higher phase velocity, and laser depletion is slower. A higher phase velocity results in an increase in the acceleration length before electrons dephase (i.e. outrun the accelerator). For both dephasing and depletion the accelerator length increases as $L \propto n_{e}{ }^{-3 / 2}$ [11], and so the need for guiding becomes even more acute at lower densities.

High intensity laser interactions over distances longer than $z_{R}$ can be achieved by employing external guiding structures or by making use of the non-linear selffocussing effect in plasmas. External guiding structures involve imposing a transverse refractive index profile which acts as to provide a focussing force to the laser spot, typically capillary guiding [12] or a plasma channel formed by high-voltage discharges [13-15]. Guiding over distances approaching $10 \mathrm{~cm}$ has been demonstrated $[16,17]$. However, while external guiding structures often offer better pulse guiding, self-guided wakefield acceleration is attractive due to its simplicity and ease of diagnosis [18-20].

Self-guiding can be achieved if self-focussing [21, 22], resulting from the plasma response to the laser pulse itself, balances diffraction of the laser. An envelope equation for the self-guided beam radial size is given by [23]:

$$
\frac{\mathrm{d}^{2} R}{\mathrm{~d} z^{2}}+\frac{\left\langle K^{2} \tilde{r}^{2}\right\rangle}{R}-\frac{\epsilon^{2}}{R^{3}}=0,
$$

where $R^{2}=\left\langle\tilde{r^{2}}\right\rangle$ is the RMS spot size and $z$ is propagation distance. The second term represents self-focussing with $K^{2} \propto n_{e}$ being an effective focussing strength. The third term arises from diffraction with $\epsilon=\lambda_{0} / \pi$. Self-guiding takes place if the diffraction term is bal- 
anced by the self-focussing, and occurs when the laser power exceeds the critical power for self-focussing $P_{c}=$ 17.4 $\left(\omega_{0} / \omega_{p}\right)^{2} \mathrm{GW}$.

For a given laser strength $a_{0}$, the spot size when guided scales as $R_{0} \propto \lambda_{p} \equiv 2 \pi c / \omega_{p} \propto n_{e}{ }^{-1 / 2}$. Attempting to focus to smaller dimensions, though increasing the selffocussing effect, leads to beam break-up (filamentation) [24]. Hence to self-guide effectively at lower densities, not only are higher powers required, but it is also necessary to have larger spot sizes to better match the matched spot size.

While self-guiding over distances much longer than the Rayleigh range has been demonstrated [25-27], it is important to explore guiding performance over longer lengths at lower plasma densities. For example, to realise a multi-GeV self-guided laser wakefield accelerator driven by a $\lambda=800 \mathrm{~nm}$ laser, plasma densities below $n_{e} \lesssim 2 \times 10^{18} \mathrm{~cm}^{-3}$ need to be employed. Characterising self-guiding performance at such low plasma densities is essential for the design of future accelerators operating in this regime.

\section{EXPERIMENTAL RESULTS}

The experiments were conducted on the Gemini laser system at the Rutherford Appleton Laboratory. A linearly polarised laser pulse with temporal FWHM intensity duration of $45 \mathrm{fs}$, centred at $800 \mathrm{~nm}$, was focussed into a steady state gas cell. The design of the plasma cell length allowed its length to be changed in the ranges of $L_{\text {cell }}=3 \ldots 42 \mathrm{~mm}$ and $L_{\text {cell }}=50 \ldots 90 \mathrm{~mm}$. Two different focussing setups were used to investigate the self-guiding performance. The $f / 20$ focussing consisted of an off-axis parabola with $3 \mathrm{~m}$ focal length; the $f / 40$ focussing geometry comprised of a $6 \mathrm{~m}$ spherical mirror. A deformable mirror was inserted into the beam line before the focussing optic and was used to compensate for wavefront errors. In both cases, the laser focal plane was set to be at the start of gas cell.

The general schematic layout of the conducted experiments is shown in Figure 1 and is identical for both focussing optics. Spherical mirror S1 with a focal length of $f=2.5 \mathrm{~m}$ for the $f / 20$ and $f=3.175 \mathrm{~m}$ for the $f / 40$ case is used to collimate the laser beam after exiting the

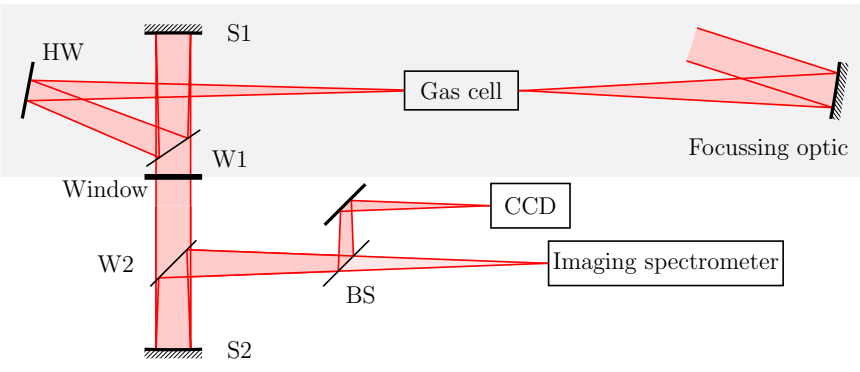

FIG. 1. Schematic depicting the experimental setup. plasma and reflecting off a wedge with a central hole HW and wedge W1. The collimated beam is transported through a high quality window to outside the vacuum chamber. The beam is refocussed with spherical mirror S2, which in conjunction with S1 forms an imaging telescope. A beamsampler BS is used to reflect part of the beam onto a 16-bit charge coupled device (CCD) with a $10 \times$ microscope objective to measure the spatial mode of the laser. The portion transmitted through BS was used to record the spatially resolved spectrum of the exiting laser pulse with an imaging spectrometer.

\section{A. f/20 results}

We first discuss experimental results obtained with the $f / 20$ focussing geometry. The elliptical laser spot waist size at $1 / e^{2}$ intensity radius, corresponding to $1 / e$ radius of the field, was (17.8 \pm 0.6$) \mu \mathrm{m}$ and $(15.8 \pm 0.5) \mu \mathrm{m}$ for the major and minor axes, respectively; the measured focal spot is shown in Figure 2a. The laser energy after compression was $\mathcal{E}_{L}=(13.5 \pm 0.8) \mathrm{J}$ and the $1 / e^{2}$ contour of the central spot contained $\alpha=$ $(55 \pm 2) \%$ of the total energy. The peak intensity was $I_{\text {peak }}=(2.8 \pm 0.1) \times 10^{19} \mathrm{~W} \mathrm{~cm}^{-2}$, resulting in a normalised peak vector potential in vacuum of $a_{0}=$ $3.6 \pm 0.3$.

Measured spatial modes for different plasma densities and gas cell lengths are shown in Figure 2 with the experimental conditions for these shots presented in Table I. The size of the laser nearfield for vacuum propagation over the same distance is plotted as the white dashed lines; for images where this contour is not shown, the laser would have diffracted to be larger than the area of the CCD. Note for Panels (e) and (f) the round clip limiting the unguided halo is the output aperture of the gas cell.

The Rayleigh range calculated from assuming a gaussian spot size of $w_{0}=\sqrt{w_{x} w_{y}}=16.8 \mu \mathrm{m}$ yields a value of $z_{R}=1.1 \mathrm{~mm}$. However, the laser spot actually deviates from a pure gaussian mode and thus the Rayleigh range adjusted by $M^{2}$ of the beam should be calculated [28]. $\quad M^{2}$ is a beam characterisation parameter which relates a hypothetical embedded gaussian beam to a realistic laser beam, thus allowing the full power of gaussian optics to be applied to the realistic beam [29]. The $M^{2}$ parameter can be defined for a gaussian beam with the same divergence as the real beam but with a beam parameter product $w_{0} \cdot \theta$ that is $M^{2} \geq 1$ times larger than that of the embedded gaussian. Here $\theta$ is the divergence of the beam and can be expressed as $\theta=1 / F_{n}$, where $F_{n}$ is the F-number. This gives $M^{2} \simeq \pi w_{0} /\left(\lambda F_{n}\right)$ and the Rayleigh range of the real beam is then given by $z_{R, R}=\pi w_{0}^{2} /\left(\lambda_{0} M^{2}\right)$. For an $f / 20$ optic, a value of $M^{2}=3.3$ is found, giving a Rayleigh range of $z_{R, R}=0.34 \mathrm{~mm}$. The image in Panel (f) in Figure 2 was taken with a plasma length of $L_{\text {cell }}=39 \mathrm{~mm}$. Thus, given the adjusted Rayleigh range, the shot shown 

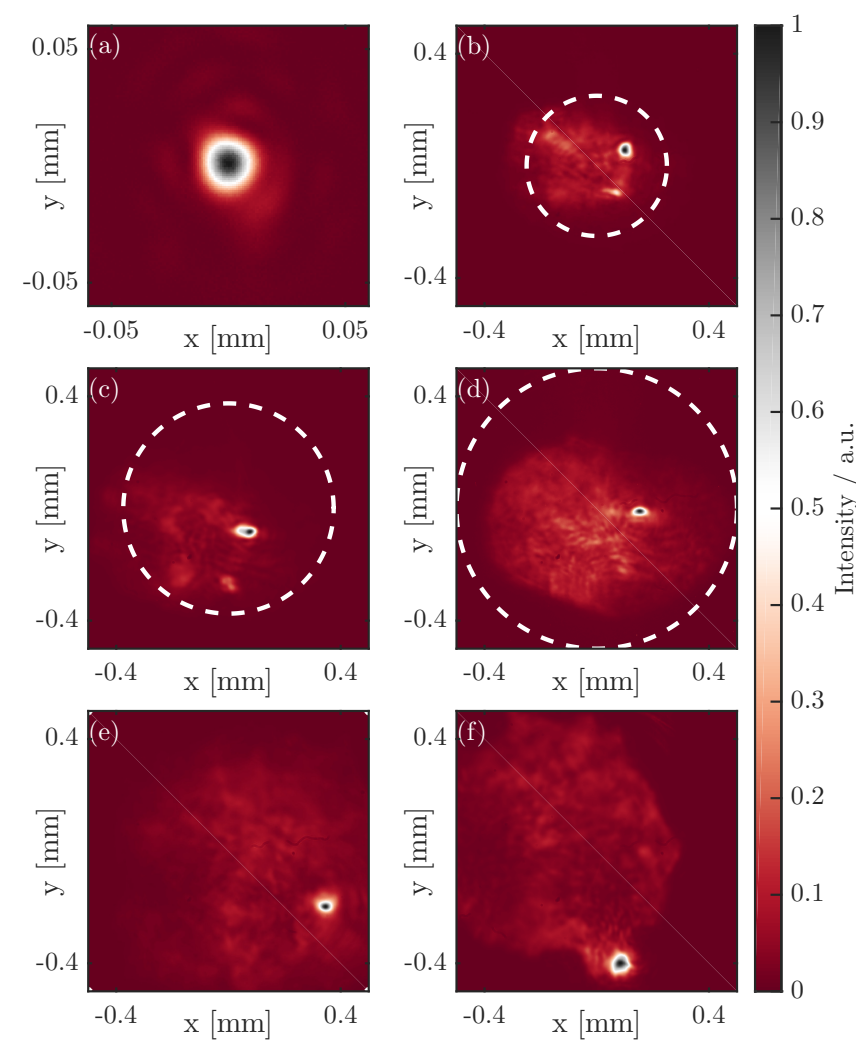

FIG. 2. Measured laser focus before entering the plasma (a) and the plane of exit from the plasma (b-f) for different gas cell lengths and plasma densities obtained with $\mathrm{f} / 20$ focussing. The white dashed line depicts the size of the laser nearfield for vacuum propagation over the same distance.

in Panel (f) in Figure 2 shows guiding for $115 z_{\mathrm{R}, \mathrm{R}}$. Assuming a gaussian input mode of the same size, the total guiding distance would be $35 z_{\mathrm{R}}$.

The measured sizes of the exiting guided modes are plotted against the plasma density these shots were taken at in Figure 3. Three different scalings are also plotted: 3D non-linear theory by Lu et al predicts $w_{m}=$ $2 \sqrt{2}\left(P / P_{c}\right)^{1 / 6} c / \omega_{p}[30]$, the envelope model by Sprangle et al predicts a spot size of $w_{m}=2 \sqrt[6]{2}\left(P / P_{c}\right)^{1 / 6} c / \omega_{p}$ [23] and the empirical scaling by Thomas et al [24] gives $w_{\mathrm{FWHM}} \simeq \lambda_{p}$, where $w_{\mathrm{FWHM}}$ is the full-width-at-halfmaximum size of the spot and $\lambda_{p}=2 \pi c / \omega_{p}$ is the wave-

\begin{tabular}{lcccc}
\hline $\begin{array}{c}\text { Panel } \\
L_{\text {cell }} \\
\mathrm{mm}\end{array}$ & $\begin{array}{c}n_{e} \\
10^{18} \mathrm{~cm}^{-3}\end{array}$ & $P / P_{c}$ & $\begin{array}{l}w_{\text {fil }} \\
\mu \mathrm{m}\end{array}$ \\
\hline (b) & 10 & 3.0 & 26 & 15.6 \\
(c) & 15 & 1.8 & 15 & 17.4 \\
(d) & 20 & 2.4 & 20 & 13.2 \\
(e) & 28 & 2.1 & 18 & 15.4 \\
(f) & 39 & 1.5 & 13 & 23.8 \\
\hline
\end{tabular}

TABLE I. Experimental parameters for exit mode images presented in Figure 2. The filament size quoted is the $1 / e^{2}$ intensity radius.

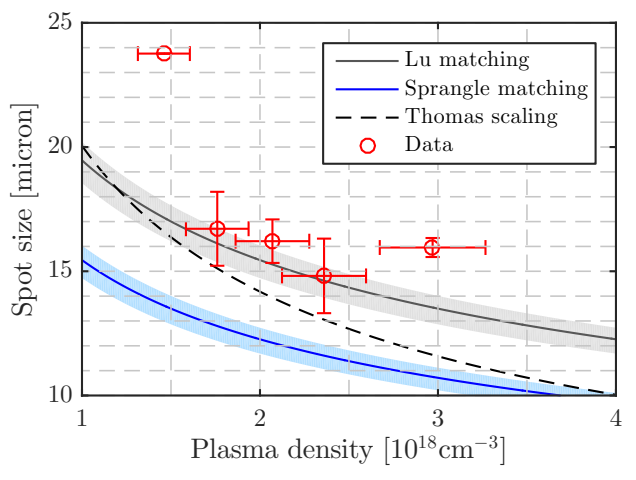

FIG. 3. Measured sizes of the self-guided laser modes as a function of plasma density, plotted for multiple shots taken at the same experimental conditions as the single shots depicted in Figure 2. Spot size corresponds to the $1 / e^{2}$ intensity radius of the spot and the data points were taken at different gas cell lengths, as shown in Table I.

length of a relativistic plasma wave. The laser power $P=\alpha P_{L} \simeq 140 \mathrm{TW}$ is used in the former two of these scalings, with $\alpha$ being the fraction of laser energy contained within the $1 / e^{2}$ intensity radius of the spot. Additionally, bands depicting a very conservative, $25 \%$ error in initial laser power are also shown. Of the presented models, the datapoints are observed to roughly agree with the scaling from the 3D non-linear theory by $\mathrm{Lu}$ et al.

\section{B. f/40 results}

With the $f / 40$ focussing geometry, the laser focal spot size at $1 / e^{2}$ intensity radius was $r_{\text {minor }}=(37 \pm 2) \mu \mathrm{m}$ and $r_{\text {major }}=(48 \pm 6) \mu \mathrm{m}$. The focal spot obtained is shown in Figure 4a. The laser energy on target was $\mathcal{E}_{L}=(8.6 \pm 0.4) \mathrm{J}$ and the $1 / e^{2}$ contour of the central spot was measured to contain $(61 \pm 6) \%$ of the laser energy. The peak intensity was calculated to be $I=(5.7 \pm 0.8) \times 10^{18} \mathrm{~W} \mathrm{~cm}^{-2}$, yielding $a_{0}=1.6 \pm 0.1$.

Figure 4 depicts single shot images of the exit plane of the plasma for different gas cell lengths. Details of the experimental conditions for each of the shots are presented in Table II. The longest gas cell length used was $L_{\text {cell }}=90 \mathrm{~mm}$, with the exiting spatial mode shown in Panel (b) in Figure 4. Again assuming a gaussian spot of $w_{0}=\sqrt{w_{x} w_{y}}=42 \mu \mathrm{m}$ yields a Rayleigh range of $z_{R}=6.9 \mathrm{~mm}$ and thus guiding over $13 z_{R}$ is observed. For the $f / 40$ optic the value $M^{2}=4.1$ is calculated, yielding $z_{\mathrm{R}, \mathrm{R}}=1.7 \mathrm{~mm}$ and thus giving guiding over $53 z_{\mathrm{R}, \mathrm{R}}$. The plasma density for this shot was $n_{e} \simeq 9.5 \times 10^{17} \mathrm{~cm}^{-3}$, giving an estimated depletion distance $L_{\mathrm{dpl}}=c \tau_{\mathrm{FWHM}}\left(\omega_{0} / \omega_{p}\right)^{2} \sim 25 \mathrm{~mm}[30,31]$. The guided filament is clearly distinguishable after propagation distance more than three times as long as the depletion length. Self-guiding is hence witnessed for distances 

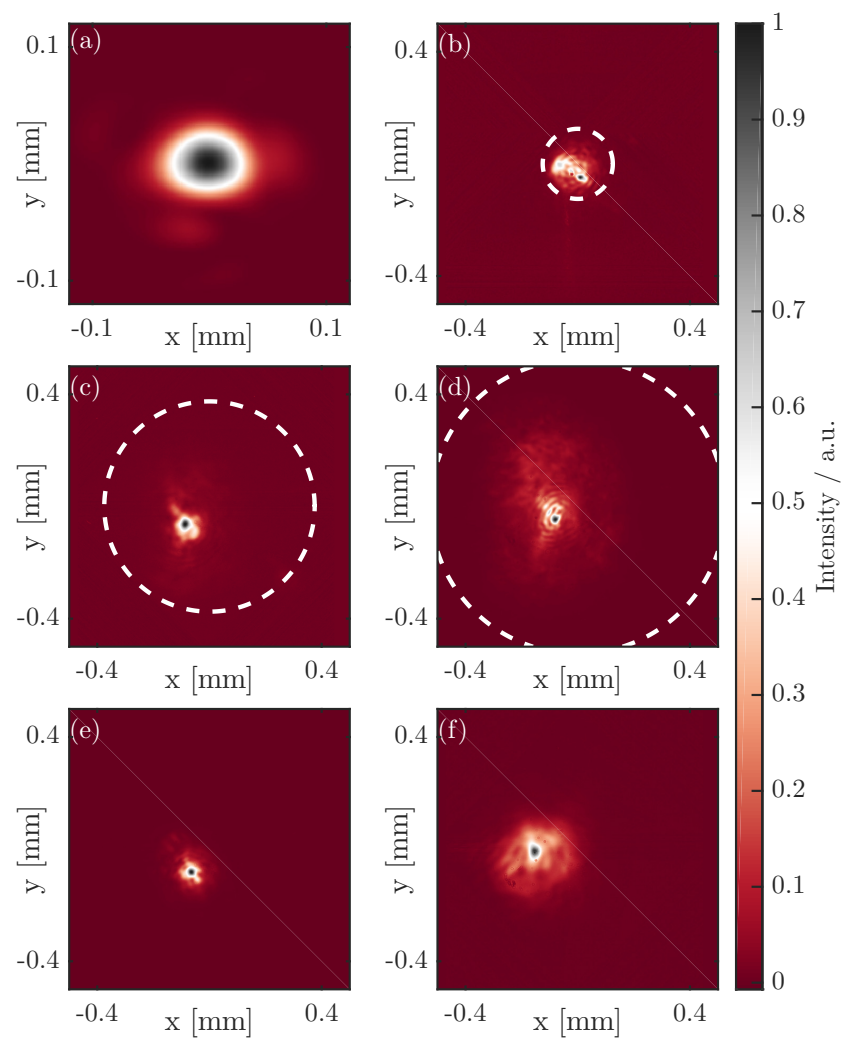

FIG. 4. Measured laser focus from $f / 40$ geometry before entering the plasma (a) and the plane of exit from the plasma (b-f) for different gas cell lengths and plasma densities. The white dashed line depicts the size of the laser nearfield for vacuum propagation over the same distance.

\begin{tabular}{lcccc}
\hline Panel & $\begin{array}{c}L_{\text {cell }} \\
\mathrm{mm}\end{array}$ & $\begin{array}{c}n_{e} \\
10^{18} \mathrm{~cm}^{-3}\end{array}$ & $P / P_{c}$ & $\begin{array}{l}w_{\text {fil }} \\
\mu \mathrm{m}\end{array}$ \\
\hline (b) & 10 & 1.5 & 9.9 & 14.3 \\
(c) & 30 & 1.3 & 8.6 & 24.2 \\
(d) & 42 & 1.6 & 11 & 16.0 \\
(e) & 70 & 1.0 & 6.6 & 16.6 \\
(f) & 90 & 0.95 & 6.3 & 24.4 \\
\hline
\end{tabular}

TABLE II. Experimental parameters for exit mode images presented in Figure 4.

much longer than depletion length. It is noticeable that the guided mode is much closer to the expected axis of propagation of the centre of the beam for all of the $f / 40$ shots.

Figure 5 depicts the beam radius at $1 / e^{2}$ intensity sizes of the laser spatial mode after exiting from a plasma with $L_{\text {cell }}=60 \mathrm{~mm}$ as a function of plasma density. Again the scalings by Lu et al, Sprangle et al and Thomas et al are plotted; the laser power $P=\alpha P_{L} \simeq 120 \mathrm{TW}$ is used in the former two of these. $25 \%$ error bands for initial laser power are also shown. While a general trend of inverse variation of the mode size with plasma density is witnessed, this does not follow any of the theoretical predictions closely. For all the plasma densities investigated

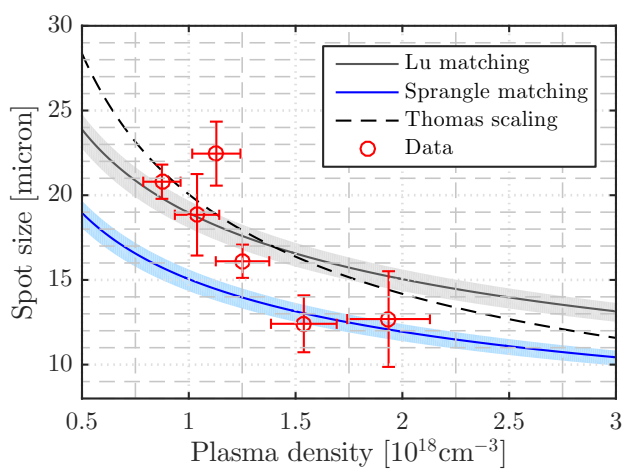

FIG. 5. Variation of the measured exit mode size with plasma density for $f / 40$ focussing optic with a $60 \mathrm{~mm}$ long gas cell. The different scalings are discussed in the main text.

here, the depletion length $L_{\mathrm{dpl}}=c \tau_{\mathrm{FWHM}}\left(\omega_{0} / \omega_{p}\right)^{2}$ is shorter than the plasma length. Thus, while self-guiding is observed at different plasma densities for a plasma length of $60 \mathrm{~mm}$, the deviation of measured mode sizes from theoretical and empirical predictions may be caused due to laser depletion.

\section{DISCUSSION}

A particle-in-cell simulation campaign in 2D would offer limited quantitative information due to the differences in focussing behaviour in 2 and 3D [32]. However, as the phasefront of the laser was not measured, full three dimensional simulations to examine the physics of pulse propagation would be not particularly representative, and computationally expensive. However, a number of characteristics can help explain the behaviour of the propagation.

For data obtained with the $f / 20$ focussing geometry an interesting asymmetry where the guided filament emerges near the edge of the unguided halo is often observed. In fact, the location of the filament was found to be very stable for multiple shots taken at conditions similar to that in Panel (f) in Figure 2. This asymmetry can be explained by considering the often imperfect quasi-nearfield of the laser near the focus [33]. In the full nearfield the spatial profile of the laser exhibits a top hat nature, where in the Fraunhofer limit in the far field a confined focal spot is observed. However, in the region close to focus, at a few Rayleigh lengths away, diffraction is governed by Fresnel diffraction as the phase variation across the beam is now bigger. Thus any imperfections in the nearfield phase can play a large role determining the intensity pattern, leading to hotspots and strongly asymmetric spatial profiles. As the laser is still focussing when entering the plasma, these nearfield imperfections will affect the coupling of the laser into the plasma and can thus affect the direction of propagation of the guided filament. This observation helps explain the prominence 
of filaments exiting near the edges of the unguided halo for the $f / 20$ data and the lack of such observations for the $f / 40$. The longer focal length leads to a larger Rayleigh length, hence reducing the degree of filamentation near the entrance of the plasma. This leads to much more centred exit position with respect to the unguided halo, as seen in Figure 4. However, a dedicated detail study of this effect is required to fully understand the underlying non-linear phenomena.

The mode sizes of self-guided beams obtained with the $f / 20$ are seen to best agree with the scaling from $\mathrm{Lu}$ et al [30], $w_{m}=2^{3 / 2}\left(P / P_{c}\right)^{1 / 6} c / \omega_{p}$, with only the lowest plasma density shot with the $f / 20$ optic showing a large deviation from the scaling predictions. However, for the $f / 40$ the spatial extent of the guided filaments was observed not to follow that scaling to the same extent. A reason for this deviation can be the different amount of energy coupled into the guided mode in the plasma. This variation can again be attributed to the spatial intensity profile near the focal plane. The upramp at the beginning of the gas cell can be thought of as an input coupler into a guided filament in the plasma. If the profile is filamented at the start of the plasma ramp, less energy will be captured. Conversely, a smoother quasi-nearfield profile will trap more energy into the plasma cavity, thus effectively providing a higher power (as $P \equiv \alpha P_{L}$ ). Hence, with a smooth input spatial mode, different plasma densities will provide a different input coupler and can thus maximise energy capture for a particular number density. Work is ongoing to quantify this in both simulations and with a theoretical model.

As the pulse propagates, its energy is depleted via the pulse front etching process [31]. The depletion length $L_{\mathrm{dpl}} \simeq c \tau\left(\omega_{0} / \omega_{p}\right)^{2}$ is most often defined as the length over which the FWHM of the laser pulse is etched away. This corresponds to an energy loss of $50 \%$ for a gaussian pulse and would thus reduce the power of the laser. In this work we have observed self-guiding over distances longer than the depletion length. However, experimental observations of pulse compression [34, 35] show that the reduction of total energy is compensated for by pulse compression. Thus the laser power stays high and can even be amplified [36]. Finally, near the end of the interaction, as the pulse power is finally reduced, the selffocussing provided by the plasma will become insufficient to guide the pulse and slow diffraction will occur. Only after the laser power falls below $P_{c}$ will there be no focussing response from the plasma and free diffraction will continue. Thus, for plasma lengths $L_{\text {plasma }}>L_{\text {dpl }}$ measurements of exciting mode size are likely to overestimate the actual size of the self-guided mode. This effect could explain the much larger measured mode sizes compared to theoretical predictions, as in Fig 3.

\section{CONCLUSION}

A study of self-guiding of short laser pulses in the plasma density range down to $<1 \times 10^{18} \mathrm{~cm}^{-3}$ allowing for multi-GeV energy gains was undertaken. Self-guiding over a distance of the order of 100 Rayleigh lengths was observed with an $f / 20$ focussing optic, with a single guided mode measured after propagation of $39 \mathrm{~mm}$ of plasma. The spatial mode sizes of the self-guided filaments follow the matched spot size predicted by the 3D non-linear theory by $\mathrm{Lu}$ et al [30]. Self-guiding over even longer distances was observed with an $f / 40$ focussing optic, with a single self-guided mode measured after propagation in $90 \mathrm{~mm}$ of $n_{e}=9.5 \times 10^{17} \mathrm{~cm}^{-3}$ plasma. This is the scale required for multi-GeV acceleration [17]. Hence, the demonstration of guiding over such long distances and low plasma densities opens the possibility of realising self-guided multi-GeV scale laser wakefield accelerator stages.

\section{Acknowledgements}

We wish to acknowledge support of the laser and engineering staff at the Central Laser Facility. This research was supported by STFC (ST/J002062/1, $\mathrm{ST} / \mathrm{P} 000835 / 1)$, and EPSRC (EP/I014462/1). SM was supported by the Royal Society. The authors confirm that the all data used in this study are available without restriction. Data can be obtained by contacting plasma@imperial.ac.uk.
[1] T. Tajima and J. Dawson, Phys. Rev. Lett. 43, 267 (1979).

[2] W. P. Leemans and E. Esarey, Physics Today 62, 44 (2009).

[3] S. P. D. Mangles et al., Nature 431, 535 (2004).

[4] J. Faure, Y. Glinec, A. Pukhov, S. Kiselev, S. Gordienko, E. Lefebvre, J. P. Rousseau, F. Burgy, and V. Malka, Nature 431, 541 (2004).

[5] C. G. R. Geddes, C. Toth, J. van Tilborg, E. Esarey, C. B. Schroeder, D. Bruhwiler, C. Nieter, J. Cary, and W. P. Leemans, Nature 431, 538 (2004).

[6] S. Kneip et al., Nature Physics 6, 980 (2010).
[7] N. D. Powers, I. Ghebregziabher, G. Golovin, C. Liu, S. Chen, S. Banerjee, J. Zhang, and D. P. Umstadter, Nature Photonics 8, 28 (2014).

[8] S. Chen, N. D. Powers, I. Ghebregziabher, C. M. Maharjan, C. Liu, G. Golovin, S. Banerjee, J. Zhang, N. Cunningham, A. Moorti, S. Clarke, S. Pozzi, and D. P. Umstadter, Phys. Rev. Lett. 110, 155003 (2013).

[9] G. Sarri, D. J. Corvan, W. Schumaker, J. M. Cole, A. Di Piazza, H. Ahmed, C. Harvey, C. H. Keitel, K. Krushelnick, S. P. D. Mangles, Z. Najmudin, D. Symes, A. G. R. Thomas, M. Yeung, Z. Zhao, and M. Zepf, Phys. Rev. Lett. 113, 224801 (2014). 
[10] G. Sarri, K. Poder, J. M. Cole, W. Schumaker, B. Reville, A. D. Piazza, D. Doria, L. Gizzi, G. Grittani, S. Kar, C. H. Keitel, K. Krushelnick, S. Kushel, S. P. D. Mangles, Z. Najmudin, D. R. Symes, A. G. R. Thomas, M. Vargas, and M. Zepf, Nat. Comm. 6, 1 (2015).

[11] W. Lu, C. Huang, M. Zhou, W. B. Mori, and T. Katsouleas, Phys. Rev. Lett. 96, 165002 (2006).

[12] B. Cros, C. Courtois, G. Matthieussent, A. D. Bernardo, D. Batani, N. E. Andreev, and S. Kuznetsov, Phys. Rev. E 65, 026405 (2002).

[13] A. Butler, D. J. Spence, and S. M. Hooker, Phys. Rev. Lett. 89, 185003 (2002).

[14] N. C. Lopes, G. Figueira, L. O. Silva, J. M. Dias, R. Fonseca, L. Cardoso, C. Russo, C. Carias, G. Mendes, J. Vieira, and J. T. Mendonça, Phys. Rev. E 68, 035402 (2003).

[15] C. Benedetti, C. B. Schroeder, E. Esarey, and W. P. Leemans, Phys. Plasmas 19, 053101 (2012).

[16] F. Wojda, K. Cassou, G. Genoud, M. Burza, Y. Glinec, O. Lundh, A. Persson, G. Vieux, E. Brunetti, R. P. Shanks, D. A. Jaroszynski, N. E. Andreev, C.-G. Wahlström, and B. Cros, Phys. Rev. E 80, 066403 (2009).

[17] W. P. Leemans, A. J. Gonsalves, H.-S. Mao, K. Nakamura, C. Benedetti, C. B. Schroeder, C. Tóth, J. Daniels, D. E. Mittelberger, S. S. Bulanov, J.-L. Vay, C. G. R. Geddes, and E. Esarey, Phys. Rev. Lett. 113, 245002 (2014).

[18] N. H. Matlis, S. Reed, S. S. Bulanov, V. Chvykov, G. Kalintchenko, T. Matsuoka, P. Rousseau, V. Yanovsky, A. Maksimchuk, S. Kalmykov, G. Shvets, and M. C. Downer, Nat. Phys. 2, 749 (2006).

[19] A. Buck, M. Nicolai, K. Schmid, C. M. S. Sears, A. Sävert, J. M. Mikhailova, F. Krausz, M. C. Kaluza, and L. Veisz, Nat. Phys. 7, 543 (2011).

[20] A. Sävert, S. P. D. Mangles, M. Schnell, E. Siminos, J. M. Cole, M. Leier, M. Reuter, M. B. Schwab, M. Möller, K. Poder, O. Jäckel, G. G. Paulus, C. Spielmann, S. Skupin, Z. Najmudin, and M. C. Kaluza, Phys. Rev. Lett. 115, 055002 (2015).

[21] C. Max, J. Arons, and A. Langdon, Phys. Rev. Lett. 33, 209 (1974).

[22] G.-Z. Sun, E. Ott, Y. C. Lee, and P. Guzdar, Phys. Fluids 30, 526 (1987).

[23] P. Sprangle, C.-M. Tang, and E. Esarey, IEEE Trans. Plas. Sci. 15, 145 (1987).

[24] A. G. R. Thomas, Z. Najmudin, S. P. D. Mangles, C. D. Murphy, A. E. Dangor, C. Kamperidis, K. L. Lancaster, W. B. Mori, P. A. Norreys, W. Rozmus, and K. Krushelnick, Phys. Rev. Lett. 98, 095004 (2007).

[25] J. E. Ralph, K. a. Marsh, A. Pak, W. Lu, C. E. Clayton, F. Fang, W. B. Mori, and C. Joshi, Phys. Rev. Lett. 102, 175003 (2009).

[26] S. Kneip et al., Phys. Rev. Lett. 103, 035002 (2009).

[27] C. E. Clayton, J. E. Ralph, F. Albert, R. A. Fonseca, S. H. Glenzer, C. Joshi, W. Lu, K. A. Marsh, S. F. Martins, W. B. Mori, A. Pak, F. S. Tsung, B. B. Pollock, J. S. Ross, L. O. Silva, and D. H. Froula, Phys. Rev. Lett. 105, 105003 (2010).

[28] S. Hooker and C. Webb, Laser Physics (Oxford University Press, 2010).

[29] A. E. Siegman, in DPSS (Diode Pumped Solid State) Lasers: Applications and Issues, Vol. 17 (OSA, Washington, D.C., 1998) p. MQ1.
[30] W. Lu, M. Tzoufras, C. Joshi, F. S. Tsung, W. B. Mori, J. M. Vieira, R. A. Fonseca, and L. O. Silva, Phys. Rev. ST Accel. Beams 10, 061301 (2007).

[31] C. D. Decker, W. B. Mori, K.-C. Tzeng, and T. Katsouleas, Phys. Plasmas 3, 2047 (1996).

[32] F. S. Tsung, W. Lu, M. Tzoufras, W. B. Mori, C. Joshi, J. M. Vieira, L. O. Silva, and R. A. Fonseca, Phys. Plasmas 13, 056708 (2006).

[33] B. Beaurepaire, A. Vernier, M. Bocoum, F. Böhle, A. Jullien, J. P. Rousseau, T. Lefrou, D. Douillet, G. Iaquaniello, R. Lopez-Martens, A. Lifschitz, and J. Faure, Physical Review X 5, 1 (2015), arXiv:1501.05797.

[34] J. Schreiber, C. Bellei, S. P. D. Mangles, C. Kamperidis, S. Kneip, S. R. Nagel, C. A. J. Palmer, P. P. Rajeev, M. J. V. Streeter, and Z. Najmudin, Phys. Rev. Lett. 105, 235003 (2010).

[35] J. Faure, Y. Glinec, J. Santos, F. Ewald, J.-P. Rousseau, S. Kiselev, A. Pukhov, T. Hosokai, and V. Malka, Phys. Rev. Lett. 95, 205003 (2005).

[36] M. J. V. Streeter, Ultrafast Dynamics of Relativistic Laser Plasma Interactions, Ph.D. thesis, Imperial College London (2013). 